\title{
Article \\ Adsorption of Hydrolysed Polyacrylamide onto Calcium Carbonate
}

\author{
Jin Hau Lew ${ }^{1}$, Omar K. Matar ${ }^{1}{ }^{(0}$, Erich A. Müller ${ }^{1}{ }^{(}$, Myo Thant Maung Maung ${ }^{2}$ and Paul F. Luckham ${ }^{1, *}$ \\ 1 Department of Chemical Engineering, Imperial College London, London SW7 2AZ, UK; \\ s.lew20@imperial.ac.uk (J.H.L.); o.matar@imperial.ac.uk (O.K.M.); e.muller@imperial.ac.uk (E.A.M.) \\ 2 PETRONAS Research Sdn. Bhd., Bandar Baru Bangi 43000, Selangor, Malaysia; \\ maungmyothant@petronas.com \\ * Correspondence: p.luckham01@imperial.ac.uk
}

check for updates

Citation: Lew, J.H.; Matar, O.K.; Müller, E.A.; Maung, M.T.M.; Luckham, P.F. Adsorption of Hydrolysed Polyacrylamide onto Calcium Carbonate. Polymers 2022, 14, 405. https://doi.org/10.3390/ polym14030405

Academic Editors: How Wei Benjamin Teo, Anutosh Chakraborty and Mujib. L. Palash

Received: 15 December 2021

Accepted: 17 January 2022

Published: 20 January 2022

Publisher's Note: MDPI stays neutral with regard to jurisdictional claims in published maps and institutional affiliations.

Copyright: (C) 2022 by the authors. Licensee MDPI, Basel, Switzerland. This article is an open access article distributed under the terms and conditions of the Creative Commons Attribution (CC BY) license (https:// creativecommons.org/licenses/by/ $4.0 /)$.

\begin{abstract}
Carbonate rock strengthening using chemical techniques is a strategy to prevent excessive fines migration during oil and gas production. We provide herein a study of the adsorption of three types of hydrolysed polyacrylamide (HPAM) of different molecular weight (F3330S, 11-13 MDa; F3530 S, 15-17 MDa; F3630S, 18-20 MDa) onto calcium carbonate $\left(\mathrm{CaCO}_{3}\right)$ particles via spectrophotometry using a Shimadzu UV-2600 spectrometer. The results are compared to different adsorption isotherms and kinetic models. The Langmuir isotherm shows the highest correlation coefficient $\left(R^{2}>0.97\right)$ with equilibrium parameters $\left(R_{L}\right)$ ranging between 0 and 1 for all three HPAMs, suggesting a favorable monolayer adsorption of HPAM onto $\mathrm{CaCO}_{3}$. The adsorption follows pseudo-second order kinetics, indicating that the interaction of HPAM with $\mathrm{CaCO}_{3}$ is largely dependent on the adsorbate concentration. An adsorption plot reveals that the amount of HPAM adsorbed onto $\mathrm{CaCO}_{3}$ at equilibrium increases with higher polymer molecular weight; the equilibrium adsorbed values for F3330S, F3530S and F3630S are approximately $0.24 \mathrm{mg} / \mathrm{m}^{2}, 0.31 \mathrm{mg} / \mathrm{m}^{2}$, and $0.43 \mathrm{mg} / \mathrm{m}^{2}$, respectively. Zeta potential analysis shows that $\mathrm{CaCO}_{3}$ has a zeta potential of $+12.32 \mathrm{mV}$, which transitions into negative values upon introducing HPAM. The point of zero charge (PZC) is observed at HPAM dosage between 10 to $30 \mathrm{ppm}$, in which the $\mathrm{pH}$ here lies between 9-10.
\end{abstract}

Keywords: polymer adsorption; kinetics; adsorption isotherm; polyacrylamide; calcium carbonate

\section{Introduction}

Carbonate reservoirs are ubiquitous in the oil and gas industry as they contribute to approximately $60 \%$ of the global petroleum reserves and provide lucrative potential for additional gas reserves [1,2]. However, much of the global hydrocarbon reserves are often found in poorly consolidated reservoirs [3] which have a relatively young geological age. For this reason, the rock grains have not undergone sufficient natural cementation by mineral deposition; hence, they often have weak and unconsolidated structures [4]. Reservoirs with weak formation strength have many detrimental consequences to drawdown operation, especially in the form of fine solid production. As hydrocarbon is consistently extracted from the reservoir, there is a depletion in the pore pressure which causes an increase in the effective stress exerting onto the formation rock. Normally, if the rock structure is rigid and strong enough, the formation rock will only deform gradually. However, for highly porous rock or weak formation rocks, there is a high tendency of abnormal increase in effective stress with fluid withdrawal, leading to irreversible deformation [5-7]. As the weak rocks deform and collapse, they are crushed, leading to the production of fines, a process also known as fines migration, which may be aggravated by other factors such as high drawdown pressure, sudden collapse of formation pores and water breakthrough within reservoir [4].

In order to overcome the long-standing issue of fines production, the oil and gas industry has been developing various mitigation strategies for this purpose. These schemes can 
be generally categorized into either mechanical or chemical methods. Common mechanical fines entrapment techniques include stand-alone sand screens, gravel packs or resin-coated gravel packs. These techniques can also be coupled with one another to enhance the fine entrapment performance [8]. However, mechanical techniques are not without their limitations and disadvantages. These techniques are often more time-consuming and expensive when compared with chemical techniques, not to mention other issues including productivity reduction, complexity in installing the equipment, installation damage to the wellbore and causing interference to reservoir operation [9-11]. Therefore, chemical technique provides an attractive alternative to consolidate weak rock formation. Chemical consolidation techniques basically involve the injection of a form of reactive chemical into the loose formation to bind the loose sand grains together. The outcome of this technique is usually the increment of uniaxial compressive strength (UCS) of the formation.

Polyacrylamide (PAM), which is a commonly used polymer in Enhanced Oil Recovery (EOR), is an attractive candidate for deployment as a formation-strengthening chemical. The adsorption of PAM in oil and gas applications has been studied specifically for EOR application. Wang et al. [1] conducted an adsorption of a hydrophobic PAM onto negatively charged calcite. Their hydrophobic PAM was a copolymer of acrylamide (AM), 2-acrylamide-2-methylpropanesulfonic acid (AMPS), 2-methacryloyloxyethyl 12-alkyl dimethyl ammonium bromide (MADA) in a 1:0.35:0.05 molar ratio, and the polymer was synthesized through free radical polymerization using ammonium persulfate as initiator. According to their results, hydrophobic PAM adsorbs onto calcite via hydrogen bonding, and they exhibit a Langmuir type adsorption isotherm which indicates monomolecular PAM adsorption onto a homogeneous adsorbent. The adsorbed amount of PAM onto calcite (in terms of per gram of calcite) increases with decreasing particle size as smaller particle size corresponds to higher particle surface area. The presence of dilute salt ions also increases the amount of PAM adsorbed, as salt ions shielded the charge on the HPAM chains, contracting the polymer chains, thus enabling more polymer to adsorb. However, an increase in environment temperature decreases the adsorbed PAM amount as the formation of new surface interactions during adsorption is exothermic, thus increasing background temperature shifts the equilibrium adsorption in the opposite direction.

Peng et al. [12] studied the effect of cationic polyacrylamide (CPAM) on precipitated calcium carbonate (PCC) flocculation. They investigated the kinetics of the CPAM adsorption onto PCC, as well as the effect of CPAM charge density and background ionic strength on the adsorption. By measuring the Photometric Dispersion Analyzer Ratio (R), they were able to observe the influence of CPAM charge density and ionic strength on the final PCC flocculate size. The CPAM charge densities used were $5 \%, 10 \%$ and $40 \%$, while the ionic strength was set to be $0,0.01$ and $0.1 \mathrm{M}$. They found that the adsorption behavior of CPAM on PCC is not straightforward: in the absence of salt, electrostatic attraction towards PCC dominates in high charge CPAM, while hydrogen bonding of CPAM amide group and carboxyl group of PCC dominates in low charge density CPAM. The presence of high ionic strength essentially neutralizes the charges of the polymer, thus the flocculation behavior between polymer adsorbed PCC transitions from an electrostatic attraction to physical bridging interaction. A similar study was conducted by Rasteiro et al. [13], whereby they also studied the flocculation and adsorption of CPAM on PCC. Their results agreed with that from Peng et al. [12], where in the absence of salt, an increase in CPAM charge density, adsorption equilibrium is reached at a lower contact time, indicating high charge density favors adsorption. In addition, they reported an increase in CPAM adsorption onto PCC when the molar mass of CPAM increases. They explained that lower molar mass polymer adopts a flat configuration on adsorbent surface, thus occupying more surface space. CPAM adsorption also increases when the molecular structure of CPAM is non-linear, as the introduction of side groups in the polyelectrolyte molecule transitions the flocculation mechanism from a patching to a bridging one $[14,15]$. Essentially, highly branched polymers produce more open flocs in the system, giving more opportunities for bridging mechanisms which lead to higher adsorption rates. In terms of study of PAM adsorption 
kinetics and isotherm, Zhu et al. [16] conducted a study on the adsorption of hydrolysed PAM (HPAM) onto quartz sand surface. Their results indicate that HPAM adsorption fits a Langmuir isotherm, which agrees with several published papers [1,17-19]. HPAM adsorption kinetics also follows a pseudo-second order model, indicating that chemisorption is the main adsorption mechanism between HPAM and quartz sand surface.

Nevertheless, to the best of our knowledge, the adsorption studies of PAM are limited to cationic PAM and sandstone, with very little reported on anionic PAM or hydrolysed PAM (HPAM) adsorption onto calcium carbonate $\left(\mathrm{CaCO}_{3}\right)$. This is especially significant as calcium carbonate at $\mathrm{pH}$ below 7 is positively charged due to the presence of protonated hydrogen ions bonded to non-bridging oxygen atoms in the $\mathrm{CaCO}_{3}$ lattice structure [20]. It is instinctive to use an anionic polymer to perform adsorption onto a positively charged adsorbent. Therefore, this paper aims to investigate the adsorption kinetics and characteristics of HPAM onto positively charged $\mathrm{CaCO}_{3}$ while assessing how polymer concentration and molecular weight affect the adsorption process.

\section{Materials and Methods}

\subsection{Material and Equipment}

The HPAM used in this work were obtained from SNF Floerger (Wakefield, UK). These polymers were coded F3330S (30\% hydrolysed, 11-13 MDa), F3530S (30\% hydrolysed, 15-17 MDa), and F3630S (30\% hydrolysed, 18-20 MDa). The molecular formula of the HPAM is illustrated in Figure 1, where the negative charge of the polymer comes from the deprotonation of carboxyl groups of the acrylate monomer into carboxylate group [21]. The calcium carbonate $\left(\mathrm{CaCO}_{3}, \geq 99 \%\right)$ powder was purchased from VWR Chemicals Ltd. (Lutterworth, UK). The solvent used in this work was deionized water (DI, $18 \mathrm{M} \Omega \mathrm{Ohm}$ ). All the experiments were conducted under ambient conditions.

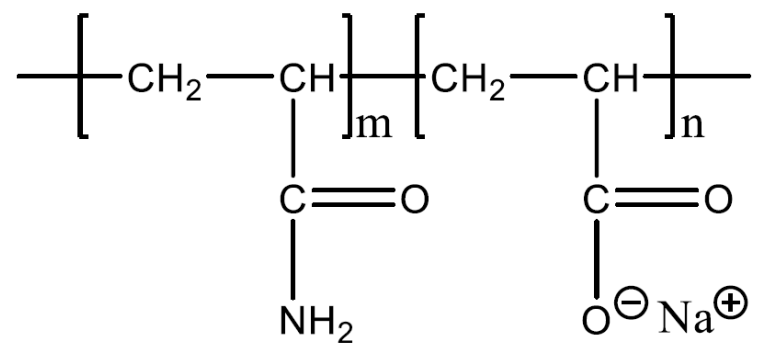

Figure 1. Molecular formula of HPAM.

The experimental equipment included a 2 mag MIX15 multi-stirrer ( 2 mag, Munich, Germany) for sample stirring, Shimadzu UV-2600 spectrophotometer (Shimadzu, Kyoto, Japan) to study the absorbance of the sample, Anton Paar Litesizer 500 (Anton Paar GmbH, Graz, Austria) to study the zeta potential of the HPAM- $\mathrm{CaCO}_{3}$ mixture, a Malvern Mastersizer 2000 (Malvern, England, UK) to measure the particle size of the $\mathrm{CaCO}_{3}$ used, a Micromeritics TriStar 3000 (Micromeritics, Norcross, GA, USA) to determine the surface area of the $\mathrm{CaCO}_{3}$ used and a Fisherbrand accuSpin 400 centrifuge (Fisher Scientific, Waltham, MA, USA) to separate the two-phases of the HPAM-CaCO 3 mixture.

\subsection{Experimental Procedure}

\subsubsection{Particle Size and Surface Area Determination}

Before commencing the adsorption analysis, the particle size of the $\mathrm{CaCO}_{3}$ was first determined using the Malvern Mastersizer 2000. A total of $1 \mathrm{~g}$ of $\mathrm{CaCO}_{3}$ was dispersed into $25 \mathrm{~mL}$ of DI water, and the suspension was added drop by drop with a dropper into cylindrical dispersion unit of the equipment. The dispersion was added until the scattering reached the suitable obscuration range of the equipment. The average particle diameter of the $\mathrm{CaCO}_{3}$ used is $3.48 \mu \mathrm{m}$.

The surface area of the $\mathrm{CaCO}_{3}$ was determined by BET surface area analysis using the Micromeritics TriStar 3000. A total of $200 \mathrm{mg}$ of $\mathrm{CaCO}_{3}$ were degassed under nitrogen at 
$80{ }^{\circ} \mathrm{C}$ for minimum $2 \mathrm{~h}$ before measuring the surface area. From the instrument, the BET surface area of the $\mathrm{CaCO}_{3}$ used was found to be $9.01 \mathrm{~m}^{2} / \mathrm{g}$.

\subsubsection{Polymer Adsorption Analysis}

To understand the effect of HPAM concentration on its adsorption onto $\mathrm{CaCO}_{3}, \mathrm{UV}-\mathrm{Vis}$ spectrometry of HPAM- $\mathrm{CaCO}_{3}$ mixtures was conducted. First, $1 \mathrm{~g}$ of $\mathrm{CaCO}_{3}$ was added into a $180 \mathrm{~mL}$ Bakelite cap glass bottle. After this, $25 \mathrm{~mL}$ of diluted polymer solutions with concentration ranging from $10 \mathrm{ppm}$ to $400 \mathrm{ppm}$ were prepared and poured into the Bakelite cap bottle with stirring. Each sample was triplicated to determine the standard deviation and standard error. The mixtures were subjected to $1 \mathrm{~h}, 6 \mathrm{~h}, 18 \mathrm{~h}, 24 \mathrm{~h}$ and $72 \mathrm{~h}$ stirring to determine the minimum amount of time required for equilibrium adsorption of HPAM onto $\mathrm{CaCO}_{3}$. After the adsorption, the mixtures were transferred into a conical bottom centrifuge tube and centrifuged at 8500 RPM for 40 min to remove the calcium carbonate particles. The supernatant was analysed using the Shimadzu UV2600 spectrophotometer at $225 \mathrm{~nm}$ (F3330S) and $210 \mathrm{~nm}$ (F3530S, F3630S) to determine the amount of HPAM adsorbed. These are the wavelengths which gives the highest relation coefficient $\left(R^{2}>0.99\right)$ on their respective calibration curve. The amount of HPAM adsorbed per unit surface area of carbonate, $Q_{e}\left(\mathrm{mg} / \mathrm{m}^{2}\right)$ was then calculated as follows:

$$
Q_{e}=\left(C_{0}-C_{e}\right) V / A
$$

where $C_{0}$ and $C_{e}$ are the initial and equilibrium concentration of the supernatant (mg/L), $V$ is the volume of polymer used $(\mathrm{L})$ and $A$ is the surface area of the carbonate $\left(\mathrm{m}^{2}\right)$. All the adsorption experiments were conducted at ambient temperature and pressure, which is $25{ }^{\circ} \mathrm{C}$ and $1 \mathrm{~atm}$, respectively.

\subsubsection{Polymer Adsorption Kinetics}

To understand the HPAM adsorption kinetics, $1 \mathrm{~g}$ of $\mathrm{CaCO}_{3}$ was added to $25 \mathrm{~g}$ of $300 \mathrm{ppm}$ F3530S, and the mixture was stirred from $1 \mathrm{~min}$ to $6 \mathrm{~h}$. The mixtures were collected at different time intervals and centrifuged, and the supernatant was analysed at $210 \mathrm{~nm}$ using the Shimadzu UV2600 spectrophotometer. The datapoints were then fitted into four different adsorption kinetic models. The first model is the pseudo-first order model [16]:

$$
\ln \left(Q_{e}-Q_{t}\right)=\ln Q_{e}+k_{1} t
$$

where $Q_{e}$ is the equilibrium PAM adsorption capacity per unit surface area of carbonate $\left(\mathrm{mg} / \mathrm{m}^{2}\right), Q_{t}$ is the PAM adsorption capacity per unit surface area of carbonate $\left(\mathrm{mg} / \mathrm{m}^{2}\right)$ at given time $t$, and $k_{1}$ is the pseudo-first order rate constant. This model assumes that the adsorption rate is directly proportional to the adsorbate concentration, is limited by the mass transfer resistance in the particle. Next, we use the pseudo-second order model

$$
\frac{t}{Q_{t}}=\frac{1}{k_{2} Q_{e}^{2}}+\frac{t}{Q_{e}}
$$

where $k_{2}$ is the pseudo-second order rate constant. This model assumes the adsorption rate is directly proportional to the square of adsorbate concentration and is limited by the adsorption mechanism. The Elovich model is next, expressed by

$$
Q_{t}=a+b \ln t
$$

where $a$ is the initial adsorption rate $(\mathrm{mg} /(\mathrm{g} \cdot \mathrm{min}))$, and $b$ is desorption constant. This model assumes the adsorption energy is not uniform and increases linearly with increasing surface coverage. At the same time, adsorption rate is not uniform, but decreases exponentially with the increasing adsorption capacity. Finally, the intraparticle diffusion model is given by

$$
Q_{t}=k_{i p} t^{0.5}+C_{e}
$$


where $k_{i p}$ is the rate constant of intra-particle diffusion kinetics $\left(\mathrm{g} /\left(\mathrm{mg} \cdot \mathrm{min}^{0.5}\right)\right) C_{e}$ is the equilibrium adsorption capacity at time $t$. If the fitted curve passes through the origin, then the intraparticle diffusion is the rate controlling step of the adsorption process [22].

\subsubsection{Isothermal Adsorption Model Analysis}

Upon obtaining the adsorption result, the experimental data points were fitted into different well-known isothermal adsorption models. The two most commonly used isotherms for adsorption experimental data fitting are the Langmuir and Freundlich isotherms [23]. The Langmuir isotherm mainly assumes a monolayer adsorption of adsorbates onto the surface of adsorbent, and maximum adsorption is achieved when the surface is completely covered. On the other hand, the Freundlich isotherm mainly assumes multilayer adsorption of adsorbates onto heterogeneous surfaces with different adsorption affinities [24]. A linear form of the Langmuir isotherm is expressed as follows:

$$
\frac{C_{e}}{Q_{e}}=\frac{1}{Q_{m} K_{L}}+\frac{C_{e}}{Q_{m}}
$$

where $Q_{m}$ is the maximum polyacrylamide adsorption capacity per unit surface area of carbonate $\left(\mathrm{mg} / \mathrm{m}^{2}\right)$ and $K_{L}$ is the Langmuir constant $(\mathrm{L} / \mathrm{mg})$.

An important feature of the Langmuir isotherm is a dimensionless constant which is called separation factor, or also known as equilibrium parameter $\left(R_{L}\right)$. It is calculated using through the following equation:

$$
R_{L}=\frac{1}{1+K_{L} C_{0}}
$$

This dimensionless separation factor is useful to evaluate how favourable an adsorption process can be. An irreversible adsorption reaction is found when $R_{L}=0$, the adsorption is favourable if $0<R_{L}<1$, it its linear if $R_{L}=1$, and unfavourable when $R_{L}>1$ [25].

The linear form of the Freundlich isotherm is expressed by the following equation:

$$
\log Q_{e}=\log K_{F}+\frac{1}{n} \log C_{e}
$$

where $K_{F}$ and $1 / n$ are the so-called Freundlich constants; $K_{F}$ represents the relative adsorption capacity of carbonate while $n$ is the degree of dependence of adsorption on the equilibrium polyacrylamide concentration.

Another interesting isothermal adsorption model to discuss is the Temkin isotherm, which is used to describe adsorption processes driven by strong intermolecular interactions, such as electrostatic interactions or ion exchange between adsorbate and adsorbent with heterogeneous surfaces [16]. We considered looking into this adsorption model as we expect the presence of an electrostatic attraction between the HPAM and $\mathrm{CaCO}_{3}$ which are oppositely charged. The linear form of the Temkin isotherm is expressed by

$$
Q_{E}=\frac{R T}{b} \ln K_{T}+\frac{R T}{b} \ln C_{e}
$$

where $b$ is a Temkin constant related to the heat of sorption $\left(\mathrm{J} \cdot \mathrm{mol}^{-1}\right)$ while $K_{T}$ is the Temkin isotherm constant $\left(\mathrm{L} \cdot \mathrm{g}^{-1}\right)$ [26].

\subsubsection{Zeta Potential Analysis}

The sediments of the HPAM- $\mathrm{CaCO}_{3}$ mixtures after centrifuged were collected and analysed for their zeta potential. A total of $0.1 \mathrm{~g}$ of the residue is dispersed and diluted in approximately $10 \mathrm{~mL}$ of DI water before conducting the zeta potential analysis in the Anton Paar Litesizer 500. This is to ensure the conductance lies within a suitable range of values. Having too low of a conductance means the ions at the slipping layer cannot move through the solution when an electric field is applied, while having too high a conductance 
will cause electrolysis of the sample. The diluted sample was transferred into an Omega Cuvette which is slotted in the instrument for analysis.

\section{Results and Discussion}

\subsection{Polymer Adsorption Analysis}

The effect of polymer molecular weight was studied by plotting a curve of adsorbed amount of polymer against polymer concentration for each HPAM used. The calibration curves for each polymer are shown in Figure S1 of the Supplementary Material. The adsorption result of different HPAM onto $\mathrm{CaCO}_{3}$ surface is shown in Figure 2 and a table of the values is in Table S1 of the Supplementary Material.

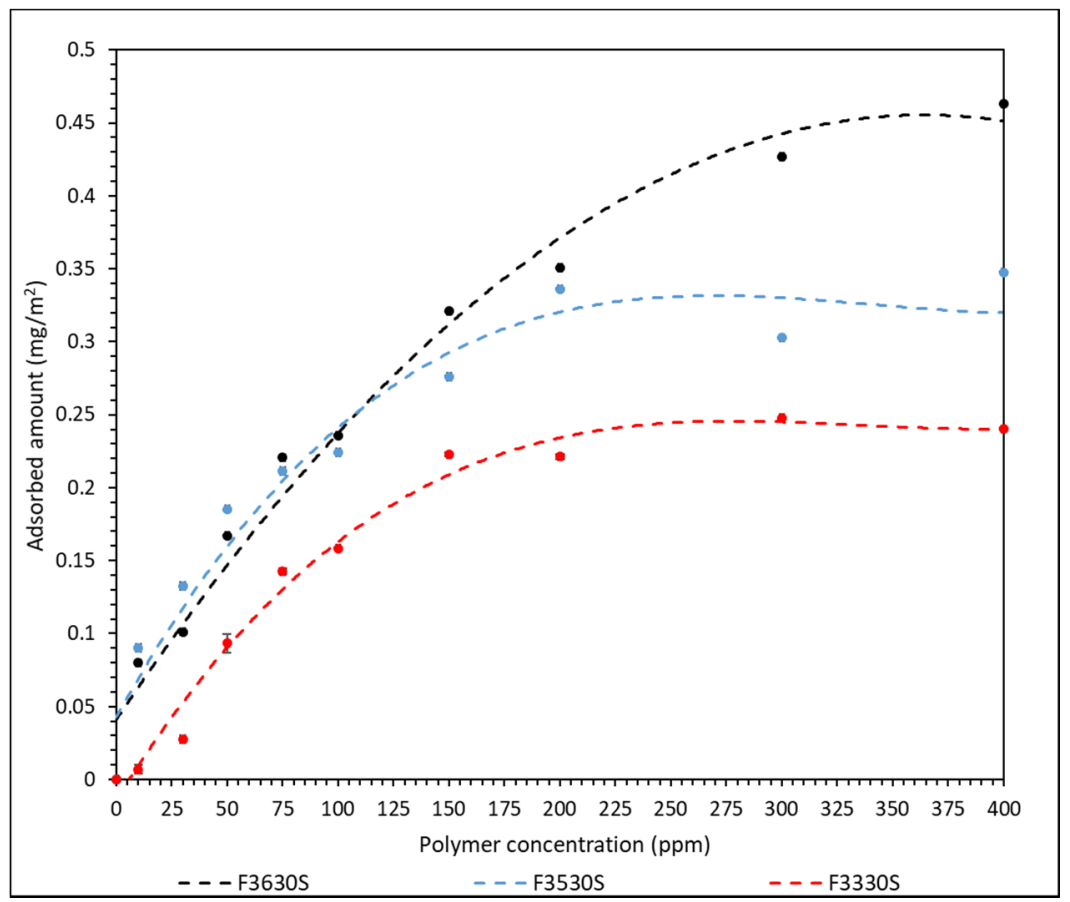

Figure 2. Amount of F3330S (11-13 MDa), F3530S (15-17 MDa) and F3630S (18-20 MDa) adsorbed onto $\mathrm{CaCO}_{3}$ against concentration of polymer dosage (Lines are a guide to the eye).

The error bars calculated from the triplicated results are incorporated into Figure 2. However, the standard error is negligible, and in most cases smaller than the individual datum point. A set of triplicated results from F3330S are included in Table S2 of the Supplementary Material as an example. From the curve of best plot in Figure 2, the adsorbed amount of both $\mathrm{F} 3330 \mathrm{~S}$ and $\mathrm{F} 3530 \mathrm{~S}$ onto $\mathrm{CaCO}_{3}$ increases steadily with increasing polymer concentration until approximately $150 \mathrm{ppm}$, where the adsorbed amount starts to plateau. F3630S has a more continuous increase in adsorbed amount where it only starts to plateau at approximately $300 \mathrm{ppm}$. Since the charge density of the HPAM used in all cases is $30 \%$, they are considered rather low charge density polymers. Hence, the polymers will mainly form extended conformation with loops and tails rather than lying flat on the $\mathrm{CaCO}_{3}$ surface. The loops and tails are responsible for the bridging interaction between different $\mathrm{CaCO}_{3}$ [27]. When a high concentration of HPAM is used, the polymers are likely to compress laterally and extend further away from the particle surface. Hence, the increasing number of loops and tails only serves to hinder late-coming polymer molecules to adsorb onto $\mathrm{CaCO}_{3}$ surface, thus the adsorbed amount of polymer will stay constant after an optimum polymer concentration is reached [28].

As observed in Figure 2, F3630S, which is the HPAM with the highest molecular weight, achieved the highest equilibrium adsorbed amount onto $\mathrm{CaCO}_{3}$, while the equilibrium value decreases with decreasing polymer molecular weight, from F3530S to F3330S. This is in line with the findings from Rasteiro et al. [13], where they also noticed a similar pattern 
in cationic PAM adsorption onto $\mathrm{CaCO}_{3}$. Based on Figure 2, the slightly higher equilibrium adsorbed amount in high molecular weight polymer is attributed to the different type of polymer conformation on adsorbent surface upon adsorption. Lower molar mass polymer tends to adopt a flat configuration, with each section of molecules occupying a larger fraction of adsorbent surface. In the case of high molecular weight polymer, there is a higher likelihood of loops and tails formation on the particle surface, hence the polymers are not lying flat on the surface. The high coverage of low molecular weight polymer is more effective in hindering late-coming polymers than that in high molecular weight polymer [29]. A depiction of this phenomenon is illustrated in Figure 3. In our experiment, F3330S, being lower molecular mass than F3630S, simply formed loops and tails to a lesser degree than F3630S, so it has a relatively flatter configuration than its F3630S counterpart.
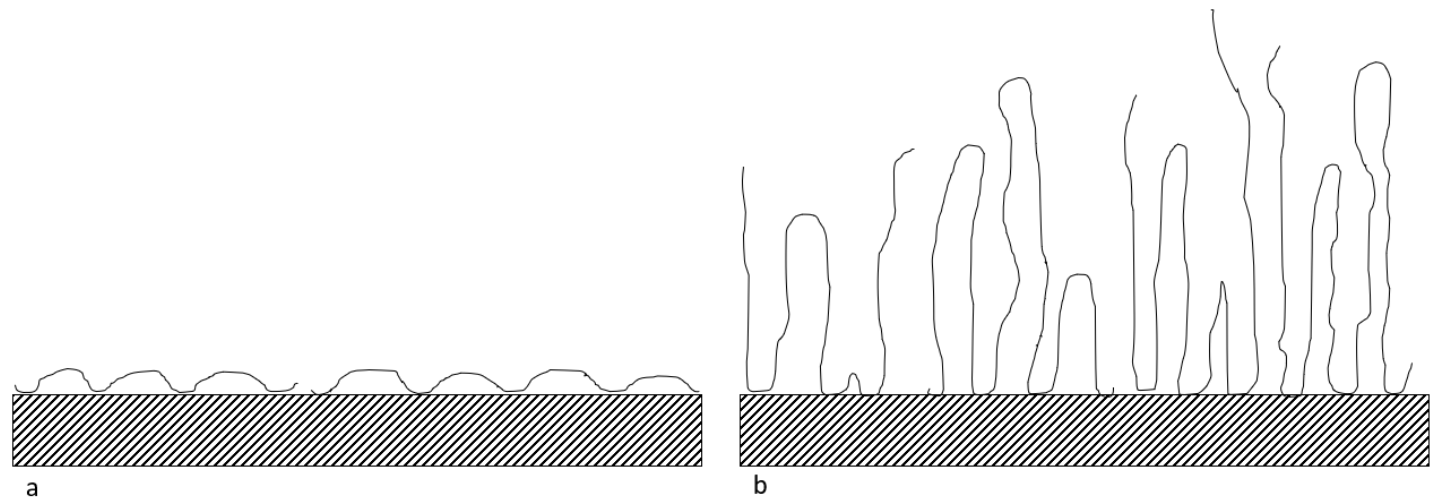

Figure 3. Configuration of (a) low molecular weight HPAM and (b) high molecular weight HPAM on the $\mathrm{CaCO}_{3}$ surface.

\subsection{Polymer Adsorption Kinetics}

The HPAM- $\mathrm{CaCO}_{3}$ mixtures were stirred for $1 \mathrm{~h}, 6 \mathrm{~h}, 18 \mathrm{~h}, 24 \mathrm{~h}$ and $72 \mathrm{~h}$ to determine the suitable period of time required to achieve maximum adsorption. This investigation is imperative when it is translated to oil and gas operation, as it signifies the amount of time required for the polymer to consolidate the reservoir properly before any hydrocarbon extraction is carried out. The adsorption results of $\mathrm{F} 3530 \mathrm{~S}$ onto $\mathrm{CaCO}_{3}$ surface over different stirring time are summarized in Table $\mathrm{S} 3$ of the Supplementary Material. The adsorption plot of F3530S onto $\mathrm{CaCO}_{3}$ over different stirring times is shown in Figure 4 .

Similar to the results presented in Figure 2, the standard deviation and error between triplicated results are very small. From the curve of best plot in Figure 4, it can be clearly observed that the adsorption has reached its maximum after $18 \mathrm{~h}$ of stirring. Below $18 \mathrm{~h}$ of stirring, the short contact time does not provide sufficient contact opportunity between the HPAM molecules and the $\mathrm{CaCO}_{3}$ particles. With increasing stirring time, the HPAM molecules have longer to adsorb onto the $\mathrm{CaCO}_{3}$ particles until a plateau is reached in the amount of adsorbed HPAM as a function of polymer concentration; this plateau is weakly dependent upon further increases in the stirring time. Hence, from Figure 4, a minimum $\mathrm{HPAM}-\mathrm{CaCO}_{3}$ contact time of $18 \mathrm{~h}$ is required for maximum amount of HPAM adsorption onto $\mathrm{CaCO}_{3}$.

The HPAM- $\mathrm{CaCO}_{3}$ mixture was also subjected to stirring from $1 \mathrm{~min}$ to $6 \mathrm{~h}$, and the supernatant was analysed with the Shimadzu UV2600 spectrophotometer. The data points from spectrometry were then fitted into different kinetic models to better understand the adsorption kinetics of HPAM onto $\mathrm{CaCO}_{3}$. The best-fitting kinetic model, which is the pseudo-second order model, is plotted in Figure 5, whereas the other two less-fitting models are shown in the Supplementary Material as Figures S2 and S3. The intraparticle diffusion model is also plotted in Figure 6. 


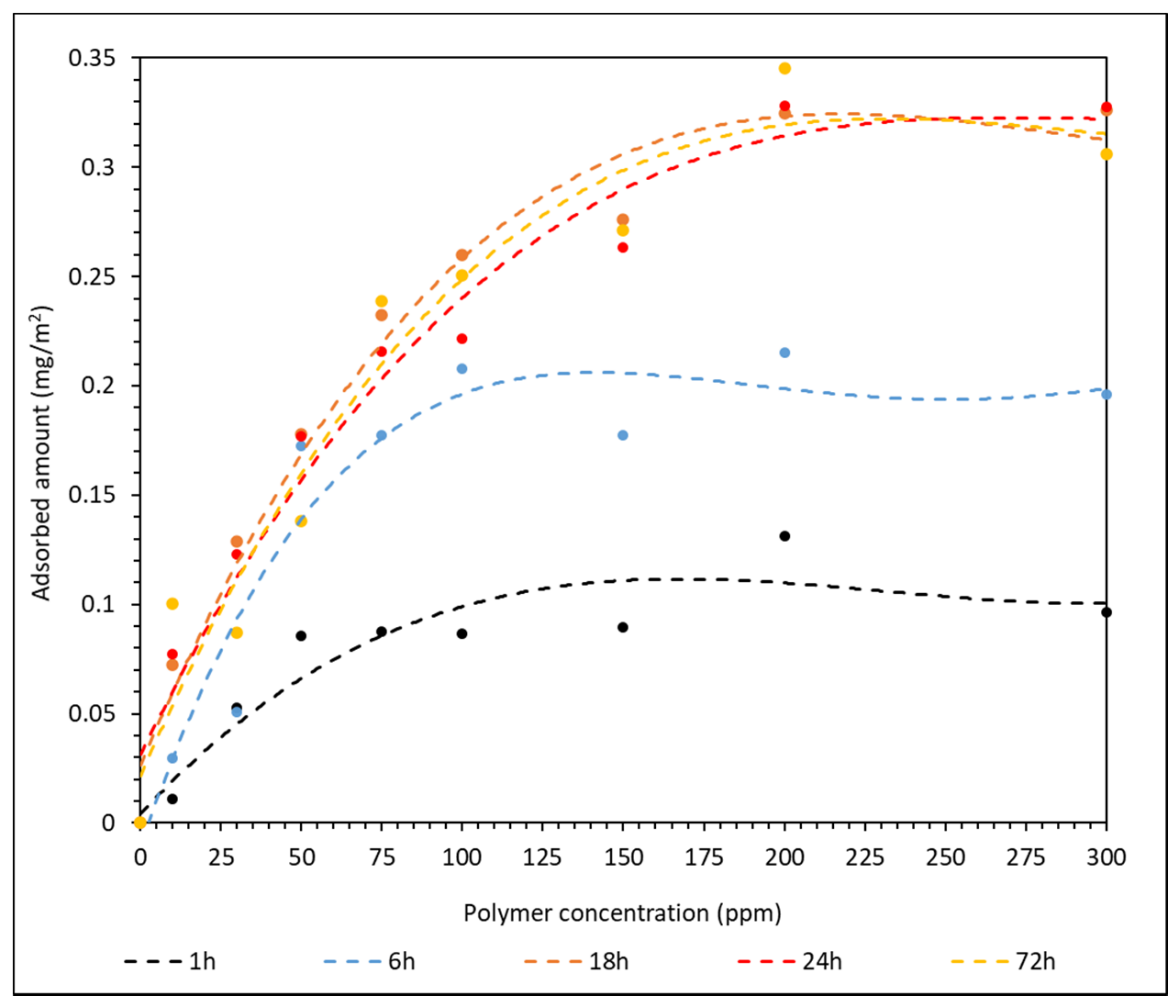

Figure 4. Effect of the stirring time on the amount of $\mathrm{F} 3530 \mathrm{~S}$ adsorbed onto $\mathrm{CaCO}_{3}$.

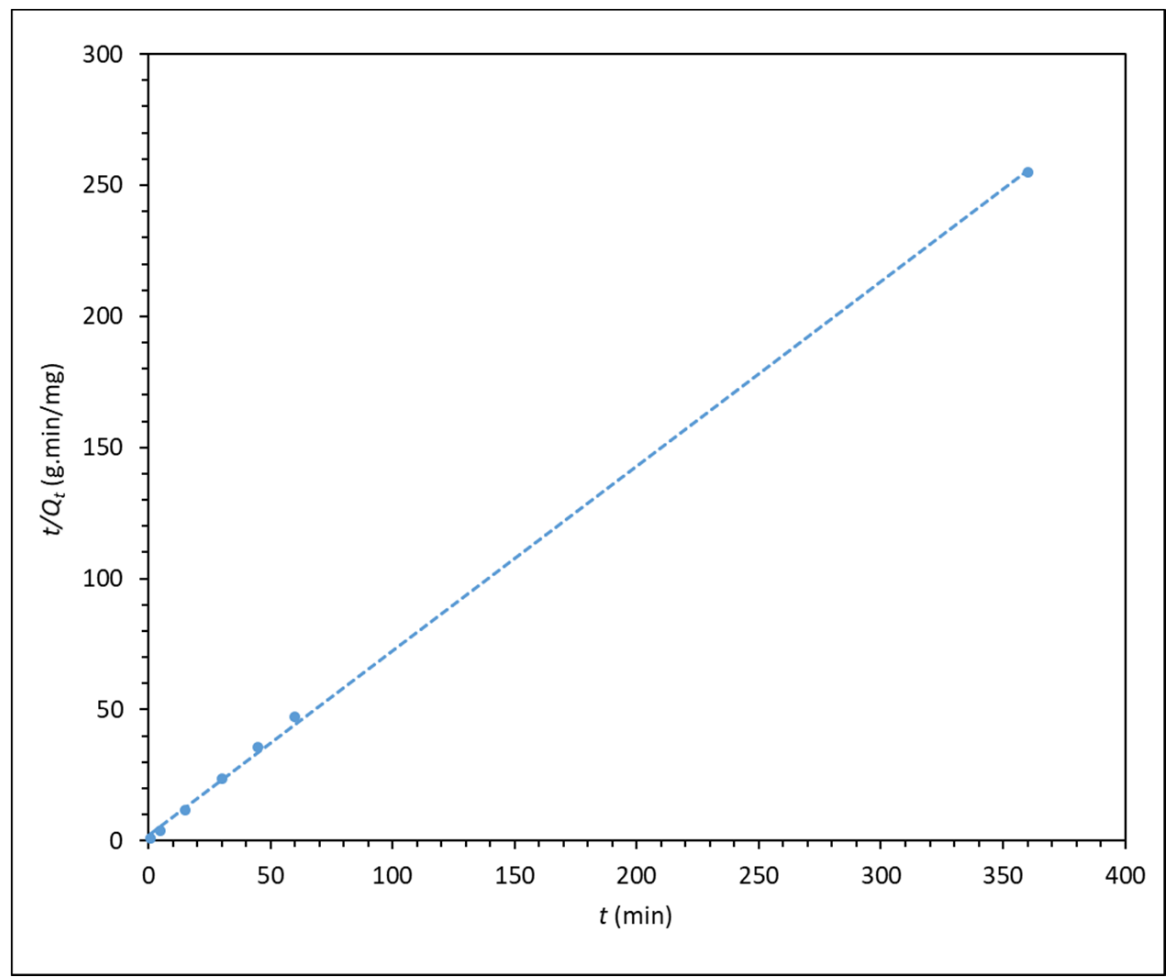

Figure 5. $t / Q_{t}$ versus time (pseudo-second order kinetic model) for the adsorption of $300 \mathrm{ppm}$ F3530S onto $\mathrm{CaCO}_{3}$. $\left(\mathrm{R}^{2}=0.9996\right)$. 


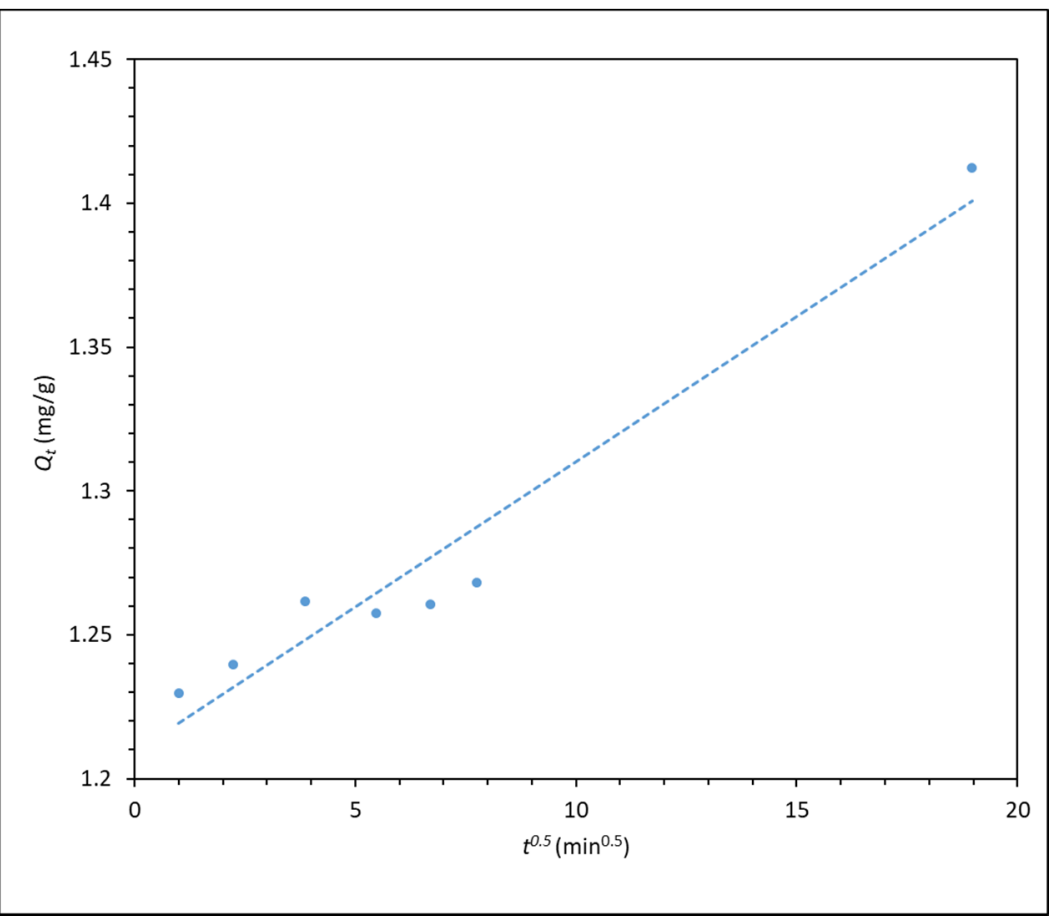

Figure 6. $Q_{t}$ versus $t_{0.5}$ (intraparticle diffusion kinetic model) for the adsorption of $300 \mathrm{ppm}$ F3530S onto $\mathrm{CaCO}_{3} \cdot\left(\mathrm{R}^{2}=0.949\right)$.

The pseudo-first and pseudo-second order kinetic plot shows very high fitting, with pseudo-second order having the highest relation coefficient $R^{2}$ of almost 1 . This shows that the pseudo-second order model best describes the adsorption kinetics of the process, indicating that the adsorption of polyacrylamide onto carbonate particle surface is largely dependent on the adsorbate concentration. This is in line with the findings from Zhu et al. [16], whereby they found that the adsorption of polyacrylamide onto a quartz sand also displayed pseudo-second order kinetics, which suggested that the adsorption process is dependent on polymer concentration, and interaction between polyacrylamide and quartz sand is mainly through electrostatic and hydrogen bonding forces. Similarly, in the case of calcium carbonate, as the $\mathrm{CO}^{-}$group of HPAM is responsible for electrostatic attraction with the surface $\mathrm{Ca}^{2+}$ ions of $\mathrm{CaCO}_{3}$ surface $[16,30,31]$. As for the intraparticle diffusion model in Figure 6, the plot shows a good degree of fitting, but it does not pass through the origin of the plot, indicating that intraparticle diffusion is not the rate-limiting step in the adsorption process. This finding is in line with that reported by Lv et al. [22], whereby the intraparticle diffusion of polyacrylamide is a rapid process, thus it cannot be the rate-limiting step.

\subsection{Isothermal Adsorption Model Analysis}

To better understand the adsorption isotherm, the adsorption data were fitted into a Langmuir, Freundlich and Temkin isotherm. The relation coefficients of each adsorption model are summarized in Table S4, and the relevant adsorption information of the Langmuir isotherm is shown in Table S5. The list of $R_{L}$ over initial concentration for each type of HPAM is tabulated in Table S6. All this information is found in the Supplementary Material. The Langmuir adsorption model fitting results for F3330S, F3530S and F3630S are shown in Figure 7, while the other Freundlich and Temkin models are shown in the Supplementary Material as Figures S4 and S5. 


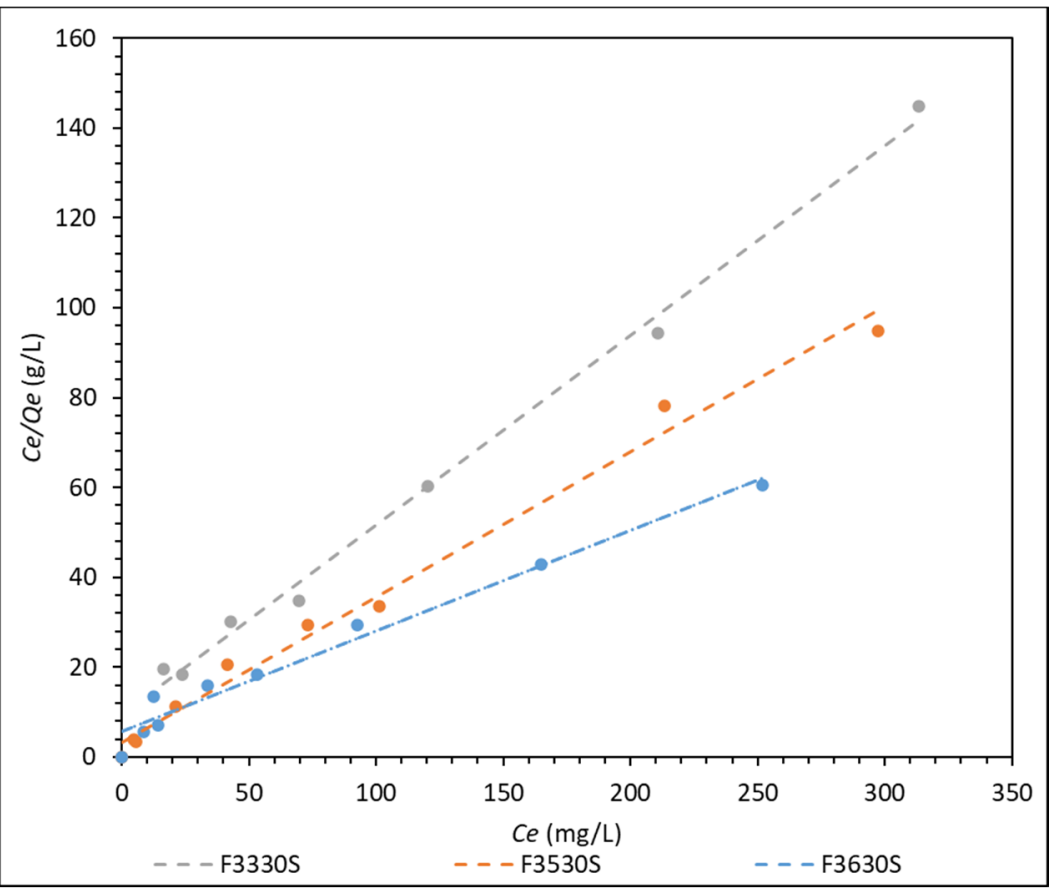

Figure 7. Langmuir adsorption isotherm model of $\mathrm{F} 3330 \mathrm{~S}, \mathrm{~F} 3530 \mathrm{~S}$ and $\mathrm{F} 3630 \mathrm{~S}$ adsorption onto $\mathrm{CaCO}_{3}$.

From Figure 7, the Langmuir adsorption isotherm shows an extremely good fit to the three HPAM solutions, with relation coefficient $R^{2}$ at approximately 0.97 and above (refer to Table S4). The same is not observed in the Freundlich and Temkin adsorption isotherm in Figures S4 and S5 where they only present a relation coefficient $R^{2}$ of between $0.65-0.89$ and $0.85-0.93$, respectively. This finding agrees with results from different literature, stating that polyacrylamide adsorbs uniformly onto the surface of the adsorbent in the form of a monolayer [1,17-19], which is often best described by the Langmuir isotherm. The HPAM molecules are adsorbed homogenously on distinct localized sites of the $\mathrm{CaCO}_{3}$ with complete coverage of these sites [32]. This could explain why the Freundlich and Temkin models do not describe HPAM adsorption well, as these two isotherms are often used to describe adsorption on a heterogenous surface.

From Table S5, it is observed that the maximum HPAM adsorption capacity per unit surface area $\mathrm{CaCO}_{3}\left(Q_{m}\right)$ is found at F3630S, which is the HPAM with highest molecular weight among the three. However, the Langmuir constant $\left(K_{L}\right)$, which reflects the affinity between adsorbent and adsorbate, is found to be the highest for F3530S [33]. These data show good agreement with the adsorption results obtained from Figure 2, as the plot does reflect highest adsorption when F3630S is used, and the $Q_{m}$ is close to the plateau values in Figure 2 .

From the tabulated $R_{L}$ values in Table 66 , all $R_{L}$ values for each polymer is between 0 and 1 regardless of polymer concentration, and the $R_{L}$ values decrease towards 0 as initial polymer concentration increases. This indicates that the adsorption of polyacrylamide onto calcium carbonate is a favorable adsorption process becoming increasingly favourable with rising polymer concentration. Amongst the three HPAM, F3530S shows the lowest overall $R_{L}$, which is in line with the highest $K_{L}$ by F3530S when compared with the lower molecular weight polymers, which shows that this particular molecular weight HPAM has the highest affinity and adsorption favor with $\mathrm{CaCO}_{3}$.

\subsection{Zeta Potential Analysis}

Zeta potential analysis is useful to understand the stability of the colloidal system. Before analysing the zeta potential of the mixture, the zeta potential of $\mathrm{CaCO}_{3}$ used was measured and the value was found to be $+12.32 \mathrm{mV}$. Each measurement was triplicated, 
and the zeta potential plot of mixture using HPAM of different molecular weight is shown in Figure 8.

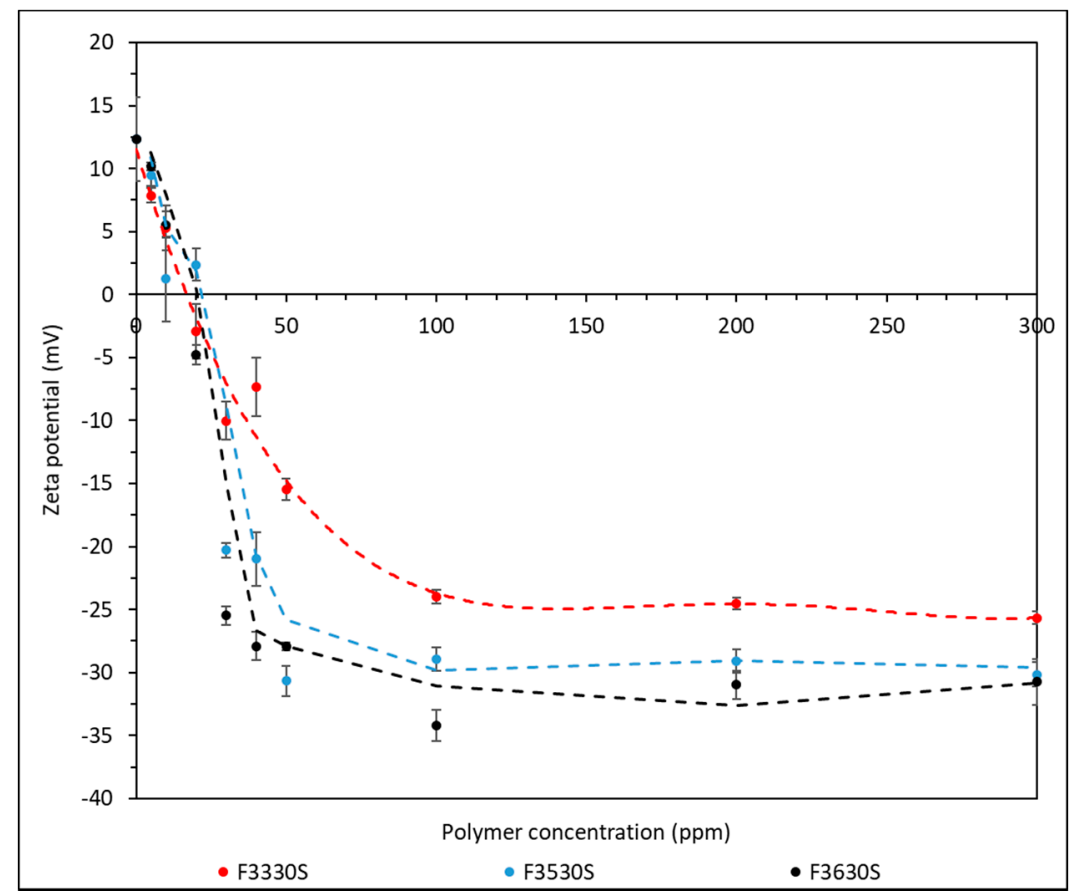

Figure 8. Zeta potential result of $\mathrm{CaCO}_{3}$ adsorbed with F3330S, F3530S and F3630S.

Based on the zeta potential results from Figure 8, all three $\mathrm{HPAM}-\mathrm{CaCO}_{3}$ mixtures showed a decrease in zeta potential with increasing polymer concentration until a plateau in zeta potential at approximately 100 ppm for F3330S, while approximately 50 ppm for F3530S and F3630S. In the absence of HPAM, steric forces between $\mathrm{CaCO}_{3}$ particles induce repulsive forces between them. As HPAM was introduced, the zeta potential decreases with increasing HPAM concentration. A transition from positive to negative charge is observed approximately between 10 to $30 \mathrm{ppm}$, in which the $\mathrm{pH}$ of the solution is between 9-10. This is in line with the point of zero charge (PZC) of 9.40-9.60 as reported [34-36]. When no HPAM is present, the repulsive forces between the carbonate particles are dominated by electrical double layer repulsion, although the zeta potential is low so that van der Waals attractive forces are likely to dominate. As more HPAM molecules are introduced, the anionic HPAM molecules will adsorb onto the positively charged carbonate particles, thus gradually neutralising the carbonate surface charge and weakening the repulsive force. After the charge reversal point, continuing to increase the HPAM dosage only builds up negative charge, which causes the force to become repulsive again. However, this repulsive force is rather weak and can be easily overcome by interparticle bridging due to polymer adsorbing simultaneously on two particles. At approximately $100 \mathrm{ppm}$ for F3330S and 50 ppm for F3530S and F3630S, the surface is said to be saturated with HPAM and the repulsive forces no longer increase [37]. Consequently, the plateau zeta potential becomes more negative with increasing polymer molecular weight. This agrees with the adsorption experiment which shows that adsorption of HPAM increases with higher polymer molecular weight. The higher HPAM concentration on $\mathrm{CaCO}_{3}$ surface induces slightly stronger anionic charge due to the carboxyl group of the polymer structure, which is then reflected as slightly more negative charge in zeta potential.

\section{Conclusions}

Hydrolysed polyacrylamides (HPAM) with different molecular weight were adsorbed onto calcium carbonate $\left(\mathrm{CaCO}_{3}\right)$ samples, and data points from spectrometry are fitted into different adsorption isotherm and kinetic models. The overall HPAM adsorption 
onto $\mathrm{CaCO}_{3}$ seems best described by a Langmuir isotherm which suggests a monolayer adsorption onto a homogeneous surface. Tabulated $R_{L}$ values show favorable adsorption of HPAM onto $\mathrm{CaCO}_{3}$, with increasing values as the initial HPAM concentration increases. The adsorption also follows a pseudo-second order kinetics indicating that the HPAM interaction with $\mathrm{CaCO}_{3}$ is largely dependent on the adsorbate concentration. The adsorbed amount of $\mathrm{F} 3330 \mathrm{~S}$ and $\mathrm{F} 3530 \mathrm{~S}$ onto $\mathrm{CaCO}_{3}$ increases with increasing HPAM concentration until it reaches a plateau at $150 \mathrm{ppm}$, which indicates the saturation of the free-binding sites on $\mathrm{CaCO}_{3}$. The same is seen for F3630S from 300 ppm onwards. The study of the different molecular weights indicates that highest equilibrium adsorbed amount of HPAM is achieved by F3630S, which is the HPAM with highest molecular weight among the three. This is due to the fact that low molecular weight polymers tend to lie flat on a particle surface, thus occupying a larger fraction of surface which hinders late-coming polymers from attaching onto the surface. Lastly, the zeta potential analysis suggests that the overall charge of the colloidal system transitions from positive to a negative charge when HPAM is introduced into a $\mathrm{CaCO}_{3}$ colloidal system.

Supplementary Materials: The following are available online at https: / www.mdpi.com/article / 10.3390/polym14030405/s1, Figure S1: Calibration curve of F3330S, F3530S and F3630S; Figure S2: $\ln \left(Q_{e}-Q_{t}\right)$ versus $t$ (pseudo-first order kinetic model) for the adsorption of $300 \mathrm{ppm}$ F3530S onto $\mathrm{CaCO}_{3}\left(\mathrm{R}^{2}=0.9869\right)$; Figure S3: $Q_{t}$ versus $\ln t$ (Elovich kinetic model) for the adsorption of 300 ppm F3530S onto $\mathrm{CaCO}_{3}\left(\mathrm{R}^{2}=0.6394\right)$; Figure S4: Freundlich adsorption isotherm model of F3330S, F3530S and F3630S adsorption onto $\mathrm{CaCO}_{3}$; Figure S5: Temkin adsorption isotherm model of F3330S, F3530S and F3630S adsorption onto $\mathrm{CaCO}_{3}$; Table S1: Adsorbed amount of F3330S, F3530S and F3630S onto $\mathrm{CaCO}_{3}$ against polymer concentration; Table S2: Example standard deviation and error from triplicated results of F3330S; Table S3: Adsorption result of F3530S over different stirring time; Table S4: Relation coefficient of adsorption model; Table S5: Adsorption information from Langmuir Isotherm; Table S6: Tabulated $R_{L}$ values.

Author Contributions: Conceptualization, O.K.M. and M.T.M.M.; methodology, P.F.L. and J.H.L.; validation, P.F.L., O.K.M. and E.A.M.; formal analysis, J.H.L.; investigation, J.H.L.; data curation, J.H.L.; writing —original draft preparation, J.H.L.; writing—review and editing, J.H.L.; supervision, P.F.L., O.K.M. and E.A.M.; funding acquisition, O.K.M. and M.T.M.M. All authors have read and agreed to the published version of the manuscript.

Funding: This project is funded by Petroliam Nasional Berhad (PETRONAS).

Institutional Review Board Statement: Not applicable.

Informed Consent Statement: Not applicable.

Data Availability Statement: Not applicable.

Acknowledgments: We acknowledge funding from PETRONAS for this work through the PETRONAS Centre for Engineering of Multiphase Systems. We also acknowledge the technical assistance provided by Patricia Carry and Kaho Cheung of the Analytical Laboratory of the Chemical Engineering Department, Imperial College London.

Conflicts of Interest: The authors declare no conflict of interest.

\section{References}

1. Wang, S.; Li, G.; Li, Y.; Guo, J.; Zhou, S.; Yong, S.; Pan, B.; Bai, B. Adsorption of new hydrophobic polyacrylamide on the calcite surface. J. Appl. Polym. Sci. 2017, 134, 45314-45321. [CrossRef]

2. Mahmood, A.; Vissapragada, B.; Alghamdi, A.H.; Allen, D.; Herron, M.; Carnegie, A.; Dutta, D.; Olesen, J.-R.; Chourasiya, R.D.; Logan, D.; et al. A Snapshot of Carbonate Reservoir Evaluation. Oilfield Rev. 2000, 12, $20-41$.

3. Nouri, A.; Vaziri, H.; Belhaj, H.; Islam, R. Effect of Volumetric Failure on Sand Production in Oil-Wellbores. In Proceedings of the SPE-Asia Pacific Oil and Gas Conference and Exhibition, Jakarta, Indonesia, 9-11 September 2003; pp. 86-93, SPE 80448.

4. Talaghat, M.R.; Esmaeilzadeh, F.; Mowla, D. Sand production control by chemical consolidation. J. Pet. Sci. Eng. 2009, 67, 34-40. [CrossRef]

5. Mowar, S.; Zaman, M.; Stearns, D.W.; Roegiers, J.C. Micro-mechanisms of pore collapse in limestone. J. Pet. Sci. Eng. 1996, 15, 221-235. [CrossRef] 
6. Soares, A.C.; Altoe, J.E.; Bedrikovetsky, P.; Ferreira, F.H. Formation Damage Due to Pore Collapse During Pressure Depletion. In Proceedings of the SPE European Formation Damage Conference, The Hague, The Netherlands, 13-14 May 2003. SPE 82254.

7. Zaman, M.; Roegiers, J.C.; Abdulraheem, A.; Azeemuddin, M. Pore Collapse in Weakly Cemented and Porous Rocks. J. Energy Resour. Technol. 1994, 116, 97-103. [CrossRef]

8. Ikporo, B.; Sylvester, O. Effect of sand invasion on oil well production: A case study of Garon field in the Niger Delta. Int. J. Eng. Sci 2015, 4, 64-72.

9. Kurawle, I.B.; Mahalle, N.A.; Kaul, M.; Nair, A.V.; Kulkarni, N.J. Silanol Resin consolidation system for deepwater completions and production optimisation. In Proceedings of the 8th European Formation Damage Conference, Scheveningen, The Netherlands, 27-29 May 2009.

10. Aggour, M.A.; Abu Khamsin, S.A.; Osman, E.S.A. A New method of Sand Control: The Process and Its First Field Implementation. In Proceedings of the SPE/IADC Middle East Drilling and Technology Conference, Amsterdam, The Netherlands, 22-24 October 2007.

11. Alakbari, F.S.; Mohyaldinn, M.E.; Muhsan, A.S.; Hasan, N.; Ganat, T. Chemical sand consolidation: From polymers to nanoparticles. Polymers 2020, 12, 1069. [CrossRef] [PubMed]

12. Peng, P.; Garnier, G. Effect of cationic polyacrylamide on precipitated calcium carbonate flocculation: Kinetics, charge density and ionic strength. Colloids Surf. A Physicochem. Eng. Asp. 2012, 408, 32-39. [CrossRef]

13. Rasteiro, M.G.; Pinheiro, I.; Ahmadloo, H.; Hunkeler, D.; Garcia, F.A.P.; Ferreia, P.; Wandrey, C. Correlation between flocculation and adsorption of cationic polyacrylamides on precipitated calcium carbonate. Chem. Eng. Res. Des. 2015, 95, 298-306. [CrossRef]

14. Rasteiro, M.G.; Garcia, F.A.P.; Ferreira, P.; Blanco, A.; Negro, C.; Antunes, E. The use of LDS as a tool to evaluate flocculation mechanisms. Chem. Eng. Processing Process Intensif. 2008, 47, 1323-1332. [CrossRef]

15. Rasteiro, M.G.; Pinheiro, I.; Garcia, F.A.P.; Ferreira, P.; Hunkeler, D. Using light scattering to screen PEL performance inflocculation. Polymers 2011, 3, 915-927. [CrossRef]

16. Zhu, S.; Ye, Z.; Liu, Z.; Chen, Z.; Li, J.; Xiang, Z. Adsorption Characteristics of Polymer Solutions on Media Surfaces and Their Main Influencing Factors. Polymers 2021, 13, 1774. [CrossRef] [PubMed]

17. Guezennec, A.-G.; Michel, C.; Bru, K.; Touzé, S.; Desroche, N.; Mnif, I.; Motelica-Heino, M. Transfer and degradation of polyacrylamide based flocculants in hydrosystems: A review. Environ. Sci. Pollut. Res. 2015, 22, 6390-6406. [CrossRef]

18. Tekin, N.; Dincer, A.; Demirbas, O.; Alkan, M. Adsorption of cationic polyacrylamide (C-PAM) on expanded perlite. Appl. Clay Sci. 2010, 50, 125-129. [CrossRef]

19. Tekin, N.; Dinçer, A.; Demirbaş, Ö.; Alkan, M. Adsorption of cationic polyacrylamide onto sepiolite. J. Hazard. Mater. 2006, 134, 211-219. [CrossRef] [PubMed]

20. Mao, Y.; Siders, P.D. Molecular Hartree-Fock model of calcium carbonate. J. Mol. Struct. Theochem. 1997, 419, 173-184. [CrossRef]

21. Laguecir, A.; Ulrich, S.; Labille, J.; Fatin-Rouge, N.; Stoll, S.; Buffle, J. Size and pH effect on electrical and conformational behavior of poly(acrylic acid): Simulation and experiment. Eur. Polym. J. 2006, 42, 1135-1144. [CrossRef]

22. Lv, Q.; Shen, Y.; Qiu, Y.; Wu, M.; Wang, L. Poly(acrylic acid)/poly(acrylamide) hydrogel adsorbent for removing methylene blue. J. Appl. Polym. Sci. 2020, 137, 49322. [CrossRef]

23. Beh, J.H.; Lim, T.H.; Lew, J.H.; Lai, J.C. Cellulose nanofibril-based aerogel derived from sago pith waste and its application on methylene blue removal. Int. J. Biol. Macromol. 2020, 160, 836-845. [CrossRef]

24. Sun, W.; Selim, H.M. Chapter Two-Fate and transport of molybdenum in soils: Kinetic modeling. In Advances in Agronomy; Sparks, D.L., Ed.; Academic Press: Cambridge, MA, USA, 2020; Volume 164, pp. 51-92.

25. Meroufel, B.; Omar, B.; Mohamed, B.; Benmoussa, Y.; Zenasni, M.A. Adsorptive removal of anionic dye from aqueous solutions by Algerian kaolin: Characteristics, isotherm, kinetic and thermodynamic studies. J. Mater. Environ. Sci. 2013, 4, 482-491.

26. Samarghandi, M.R.; Hadi, M.; Moayedi, S.; Askari, F.B. Two-parameter isotherms of methyl orange sorption by pinecone derived activated carbon. Iran. J. Environ. Health Sci. Eng. 2009, 6, 285-294.

27. Zhou, Y.; Yu, H.; Wanless, E.; Jameson, G.; Franks, G.V. Influence of polymer charge on the shear yield stress of silica aggregated with adsorbed cationic polymers. J. Colloid Interface Sci. 2009, 336, 533-543. [CrossRef] [PubMed]

28. Van De Ven, T.G.M. Kinetic aspects of polymer and polyelectrolyte adsorption on surfaces. Adv. Colloid Interface Sci. 1994, 48, 121-140. [CrossRef]

29. Lee, R.Y. Development of Sand Agglomeration Formulation for Oil \& Gas Well Applications to Reduce the Production of Fine Particulates. Ph.D. Thesis, Imperial College London, London, UK, June 2019.

30. Cui, Z.; Qi, D.; Song, B.; Pei, X.; Hu, X. Inhibiting Hydrophobization of Sandstones via Adsorption of Alkyl Carboxyl Betaines in Surfactant-Polymer Flooding Using Poly Alkylammonium Bromides. Energy Fuels 2016, 30, 2043-2051. [CrossRef]

31. Li, S.; Qiao, C.; Li, Z.; Wanambwa, S. Properties of Carbon Dioxide Foam Stabilized by Hydrophilic Nanoparticles and Hexadecyltrimethylammonium Bromide. Energy Fuels 2017, 31, 1478-1488. [CrossRef]

32. Sahu, O.; Singh, N. 13-Significance of bioadsorption process on textile industry wastewater. In The Impact and Prospects of Green Chemistry for Textile Technology; Shahidul, I., Butola, B.S., Eds.; Woodhead Publishing: Sawston, UK, 2019 ; pp. $367-416$.

33. Rezakazemi, M.; Zhang, Z. 2.29 Desulfurization Materials. In Comprehensive Energy Systems; Dincer, I., Ed.; Elsevier: Oxford, UK, 2018; pp. 944-979.

34. Farooq, U.; Tweheyo, M.T.; Sjøblom, J.; Øye, G. Surface characterization of model, outcrop, and reservoir samples in low salinity aqueous solutions. J. Dispers. Sci. Technol. 2011, 32, 519-531. [CrossRef] 
35. Sohal, M.A.; Thyne, G.; Søgaard, E.G. Review of recovery mechanisms of ionically modified waterflood in carbonate reservoirs. Energy Fuels 2016, 30, 1904-1914. [CrossRef]

36. Blackman, L.D.; Gunatillake, P.A.; Cass, P.; Locock, K.E.S. An introduction to zwitterionic polymer behavior and applications in solution and at surfaces. Chem. Soc. Rev. 2019, 48, 757-770. [CrossRef]

37. Szilagyi, I.; Trefalt, G.; Tiraferri, A.; Maroni, P.; Borkovec, M. Polyelectrolyte adsorption, interparticle forces, and colloidal aggregation. Soft Matter 2014, 10, 2479-2502. [CrossRef] 